\section{Esquistossomose mansônica no Estado do Maranhão, Brasil, 1997-2003}

\author{
Schistosomiasis mansoni in Maranhão State, \\ Brazil, 1997-2003
}

\author{
${ }_{1}$ Escola Nacional de Saúde \\ Pública Sergio Arouca, \\ Fundação Oswaldo Cruz, Rio \\ de Janeiro, Brasil. \\ Correspondência \\ A. P. Ferreira \\ Centro de Estudos da Saúde \\ do Trabalhador e Ecologia \\ Humana, Escola Nacional de \\ Saúde Pública Sergio Arouca, \\ Fundação Oswaldo Cruz. \\ Rua Leopoldo Bulhões 1480, \\ Rio de Janeiro, $R J$ \\ 21041-210, Brasil. \\ aldoferreira@ensp.fiocruz.br
}

\begin{abstract}
The aim of this study was to assess the prevalence of schistosomiasis mansoni in Maranhão State, Brazil, from 1997 to 2003, based on data from the regional health offices included in the Schistosomiasis Control Program. The statistical method was polynomial regression. The municipalities (counties) of Bacabal and São Luís showed a constantly downward trend throughout the time series. Colinas showed an increase in prevalence until 2002, followed by a downward trend. Imperatriz witnessed a constant upward trend. Socioeconomic factors are highly significant for schistosomiasis prevalence in Maranhão, which helps explain the results. The recording of data for the regional health offices is believed to have been affected by changes resulting from decentralization of the Schistosomiasis Control Program. The study's results highlight issues that extend beyond analysis of the pattern of occurrence of schistosomiasis mansoni cases in the State of Maranhão, particularly demonstrating the need for improvements in the population's living conditions.
\end{abstract}

Schistosomiasis Mansoni; Social Conditions; Prevalence
Selma Patrícia Diniz Cantanhede 1

Aldo Pacheco Ferreira 1

Ines Echenique Mattos 1

\section{Introdução}

No Brasil, a esquistossomose mansônica ocorre em 19 estados, abrangendo uma área endêmica que vai desde o Maranhão até o Espírito Santo e Minas Gerais, com focos em outros estados 1,2. Estima-se que cerca de 2.500 .000 a 8.000 .000 de brasileiros sejam portadores da doença ${ }^{3}$. Entretanto, o conhecimento acerca do comportamento epidemiológico dessa parasitose é limitado, existindo estimativas divergentes sobre sua prevalência no país 4 .

Essa parasitose possui bastante relevância no Maranhão, ocorrendo em 48 dos 217 municípios 5 . De acordo com os dados do sistema local do Programa de Controle da Esquistossomose (PCE), em 2008, houve um registro de 649 casos da doença na área de foco e 3.461 casos na área endêmica, sendo as prevalências 3,8\% e 6,7\%, respectivamente.

As atividades de vigilância e controle da doença nesse estado existem desde 1977 6. Como em demais estados do Brasil, tais atividades eram direcionadas ao tratamento dos indivíduos acometidos, enquanto o controle dos hospedeiros e as ações de saneamento e educação em saúde eram executados de modo irregular 7 . Em 1999, com a descentralização do PCE, as atividades passaram para os municípios, sendo o controle de vetores e doentes realizado por inquéritos malacológico e parasitológico, respec- 
tivamente, e havendo também a inclusão dos dados no sistema local para repasse ao sistema estadual 8 .

Em razão do exposto, este trabalho teve como objetivo analisar a prevalência da esquistossomose mansônica em população que vive em área de risco e é alvo de ações de controle do PCE no Maranhão.

\section{Material e métodos}

Trata-se de um estudo ecológico com base no Sistema de Informação do Programa de Controle da Esquistossomose (SISPCE). Analisou-se a série histórica da prevalência da esquistossomose mansônica em 15 regionais de saúde. A definição do período de estudo baseou-se na disponibilidade de dados considerados como concluídos, ou seja, situação configurada pela digitação e conferência do total de formulários.

De 1997 a 2003, o PCE relacionava como população trabalhada 9.471.299 indivíduos, tendo sido realizados 7.343.265 exames, correspondendo a 77,53\% dessa população (SISPCE; http:// www.datasus.gov.br, acessado em 10/Mar/2009). A prevalência da população trabalhada corresponde à razão entre o número de exames positivos e o número de exames realizados, multiplicado por 100. A técnica de laboratório utilizada pelo PCE é o exame parasitológico realizado com o método Kato-Katz 9 .

Analisou-se a tendência para cada regional pela utilização de modelos de regressão polinomial. A variável dependente correspondeu à prevalência anual e a variável independente, aos anos de estudo. Na modelagem, considerou-se primeiro o modelo linear, sendo posteriormente utilizados modelos mais complexos, como os de segundo e terceiro graus. A escolha do melhor modelo foi baseada na significância estatística ( $\mathrm{p} \leq 0,05)$, no valor do coeficiente de determinação $\left(\mathrm{R}^{2}\right)$ e na análise dos resíduos.

\section{Resultados}

No período de estudo foram registrados 567.192 casos $(7,42 \%)$. Bacabal e Presidente Dutra apresentaram maior prevalência, com 12,8\% e $9 \%$, respectivamente, enquanto Balsas e Pinheiro obtiveram os menores percentuais.

Quatro regionais apresentaram modelos com bom ajuste e estatisticamente significativos (Tabela 1). As curvas de tendência apresentaram comportamento desigual entre essas regionais. Bacabal e São Luís apresentaram tendência decrescente e constante (Figura 1). Em média, houve, a cada ano, declínio de 1,7 e 0,656 casos de esquistossomose, respectivamente.

A regional de Colinas demonstrou tendência de incremento até 2002, com diminuição posterior da prevalência. Em Imperatriz, encontrou-se tendência crescente e constante, sendo que, em média, ocorreu incremento de casos de esquistossomose na ordem de 0,415 a cada ano (Figura 2).

Para as demais regionais de saúde foi verificada a ausência de tendência temporal significante, ou seja, ausência de modificação significativa dos patamares do número de casos registrados ao longo do período analisado.

\section{Discussão}

Da mesma forma que outras fontes de informações secundárias, o SISPCE apresenta limitações 3,10. No caso específico, é importante ressaltar que o período 1999-2003 correspondeu à etapa pós-descentralização do PCE, quando as

Tabela 1

Modelos de regressão * dos casos de esquistossomose mansônica, segundo as regionais de saúde do Programa de Controle de Esquistossomose. Maranhão, Brasil, 1997-2003.

\begin{tabular}{lcc}
\hline Regional de saúde & Modelo ** & R2 (\%) *** \\
\hline Bacabal & $12,077-1,702 x$ & 81,3 \\
Colinas & $4,401+2,594 x-0,224 x^{3}$ & 86,3 \\
Imperatriz & $5,014+0,415 x$ & 50,4 \\
São Luís & $8,940-0,656 x$ & 77,0
\end{tabular}

* Modelos de regressão que apresentaram significância estatística;

** Modelo: $y=$ número de casos de esquistossomose mansônica $\mathrm{x}=$ ano do diagnóstico;

*** $\mathrm{R}^{2}=$ coeficiente de determinação. 
Regionais de saúde do Programa de Controle de Esquistossomose com tendência de declínio do percentual de casos de esquistossomose mansônica. Maranhão, Brasil, 1997-2003.

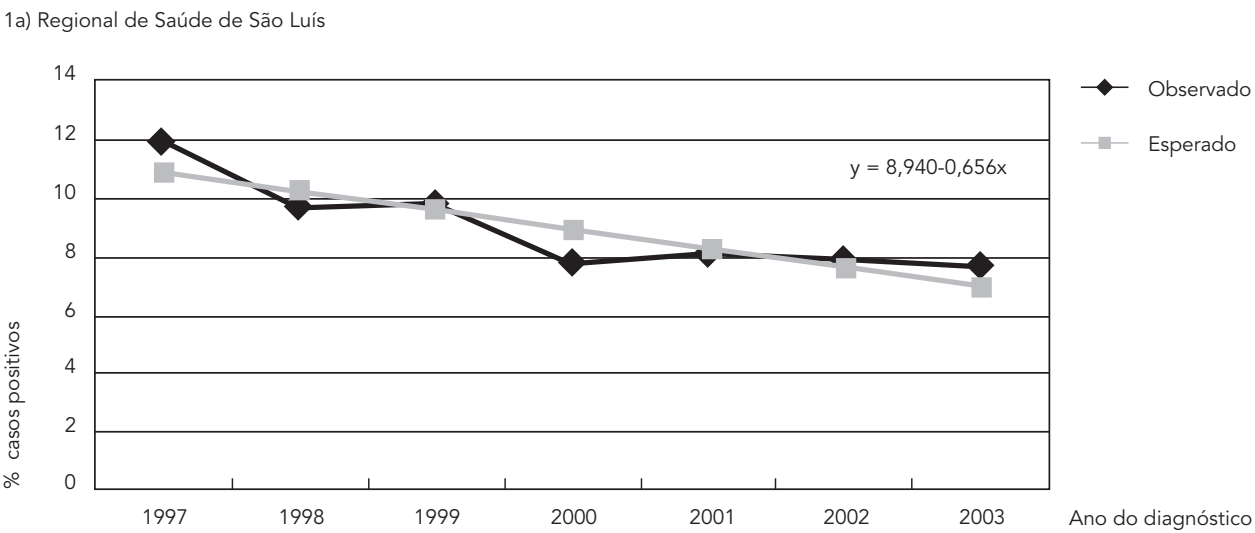

1b) Regional de Saúde de Bacabal

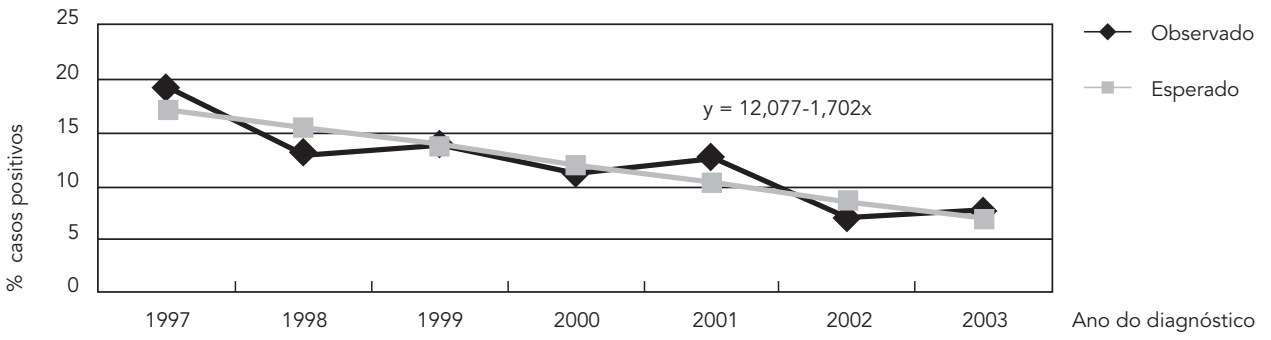

ações de controle da doença ficaram a cargo dos municípios, e cada um passou a executar as atividades de acordo com seus critérios e possibilidades 8 .

Embora as atividades de rotina do programa venham sendo executadas, os municípios têm dificuldades de exercê-las, por conta do reduzido quadro operacional e da necessidade de controle de outras doenças 8,10 .

Acredita-se que o registro de dados referente às regionais de saúde do Maranhão também tenha sido afetado pela descentralização do PCE. Entretanto, os problemas parecem não ter sido tão acentuados a ponto de mascarar os dados e inverter a configuração da série histórica das regionais de Colinas e Imperatriz. Já no caso da regional de Bacabal, as evidências apontam na direção contrária.

Nos municípios da regional de Bacabal, a proporção média de domicílios com abastecimento de água por rede geral correspondeu a $37,4 \%$, a população vivendo em pobreza equivaleu a $58,2 \%$ e a proporção média de moradias inadequadas foi de $33,8 \%$. Tendo em vista a estreita relação que todos esses aspectos apresentam com a transmissão da esquistossomose, a tendência de declínio da prevalência da doença observada nessa regional gera questionamentos, sugerindo a possibilidade de sub-registro de informações.

Em Colinas e Imperatriz, verificou-se aumento da prevalência, entre 1997 e 2003. Sugere-se que o incremento ocorrido nessas regionais esteja relacionado tanto às questões relativas ao saneamento e à pobreza, quanto aos eventos migratórios populacionais.

Outro aspecto relevante nessas regionais diz respeito ao registro de moluscos (hospedeiros intermediários). Na regional de saúde de Colinas, não existe registro de coleta para os anos de 1998 e 2001, e só há ocorrência de moluscos positivos para 1997 e 2002. Para a regional de Imperatriz, há registro de coleta de moluscos apenas para 
Regionais de saúde do Programa de Controle de Esquistossomose com tendência de incremento do percentual de casos de esquistossomose mansônica. Maranhão, Brasil, 1997-2003.

2a) Regional de Saúde de Imperatriz

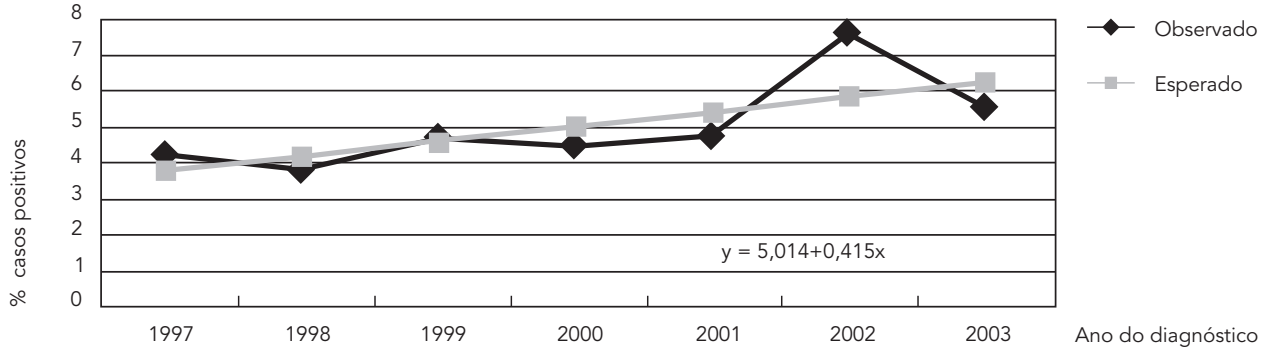

2b) Regional de Saúde de Colinas

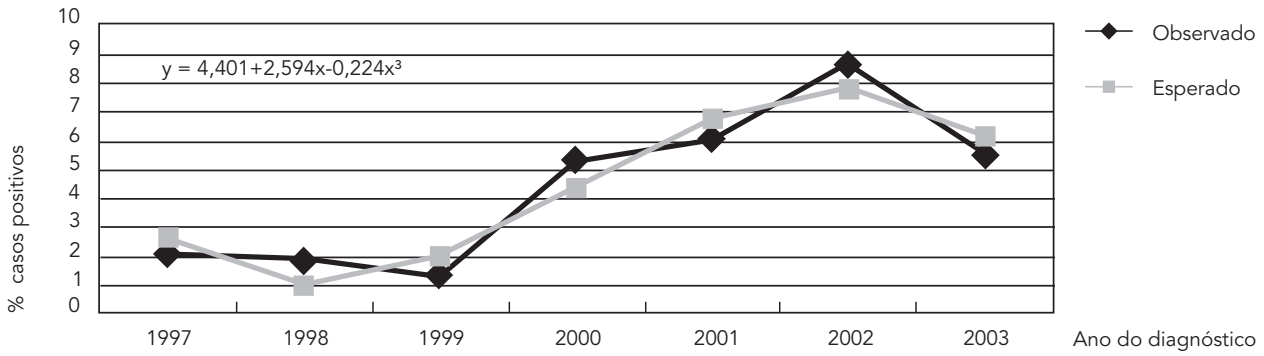

2002 e 2003, mas somente em 2002 existem dados de moluscos positivos (SISPCE; http://www. datasus.gov.br, acessado em 10/Mar/2009).

A ausência de moluscos positivos, após o ano de 2002, poderia ser apontada como uma possível explicação para o decréscimo da prevalência, ocorrido a partir desse mesmo ano, na regional de Colinas. Todavia, a interpretação das lacunas existentes no registro de moluscos deve considerar outras possibilidades, como por exemplo, a realização parcial ou a ausência de coleta para alguns anos, ou, ainda, a falta de digitação dos dados referentes às coletas. $\mathrm{O}$ declínio da prevalência nessa regional poderia também estar relacionado à negatividade dos exames, devido ao período de incubação da doença, ou a não ocorrência de reinfecções até a época de realização desses.

Cabe ressaltar que apesar de não haver registro de moluscos positivos em todos os anos, para as regionais de Colinas e Imperatriz, foi observada tendência crescente da prevalência. Tal fato reforça as hipóteses levantadas, as quais também se sustentam com base nas características socioeconômicas dos municípios que compõem essas regionais.

O baixo desenvolvimento socioeconômico e a ausência de ações de educação em saúde e de saneamento são características importantes no quadro de fatores que atuam como determinantes para a transmissão e o estabelecimento dessa parasitose 4,11 .

Quanto à tendência de declínio da prevalência na regional de São Luís, existem aspectos relevantes que sugerem justificativas plausíveis para o evento, pois a regional abriga o Município de São Luís, capital do estado e sede da estrutura política e administrativa do Maranhão, que possui melhor rede de assistência básica para o atendimento da população. Nesse sentido, é possível inferir que, devido à proximidade geográfica, os demais municípios da regional de saúde estejam sob influência do contexto socioeconômico da capital e também usufruam de benefícios, como maior cobertura de instalações sanitárias, mais oferta de serviços de saúde, 
qualidade e acesso à educação e maiores possibilidades de emprego.

Considerando os resultados obtidos e os aspectos relacionados à transmissão da esquistossomose, nosso estudo possui grande relevância, visto que demonstra a situação epidemiológica dessa parasitose num dado recorte de tempo e, ainda, sinaliza as necessidades de melhorias relacionadas às condições de vida da população.

\section{Resumo}

O objetivo deste estudo foi analisar a prevalência da esquistossomose mansônica no Maranhão, Brasil, no período de 1997 a 2003, segundo as regionais de saúde contempladas pelo Programa de Controle da Esquistossomose (PCE). O método estatístico utilizado foi a regressão polinomial. Bacabal e São Luís apresentaram tendência decrescente e constante. Colinas demonstrou tendência de incremento até o ano de 2002, com posterior diminuição. Em Imperatriz, observou-se tendência crescente e constante. Concluiu-se que as questões relativas às características socioeconômicas são bastante expressivas no Maranhão e, seguramente, possuem relação com os resultados encontrados. Acredita-se, ainda, que o registro de dados referente às regionais de saúde do estado tenha sido afetado pelas modificações ocorridas por conta da descentralização do PCE. Os resultados deste estudo apontam aspectos que transcendem a análise do padrão de ocorrência de casos de esquistossomose mansônica no Estado do Maranhão, ficando demonstrada, sobretudo, a necessidade de melhorias relacionadas às condições de vida da população.

Esquistossomose Mansoni; Condições Sociais; Prevalência
Portanto, demais investigações utilizando esse desenho de estudo também serão importantes para divulgar mais conhecimentos sobre essa doença em nosso país. Além de contribuir efetivamente para a avaliação e aprimoramento das estratégias de controle do PCE no Brasil, uma vez que esse tipo de técnica estatística, ao descrever eventos passados, viabiliza a previsão de fenômenos epidemiológicos futuros.

\section{Colaboradores}

S. P. D. Cantanhede participou da idealização e de todas as etapas de desenvolvimento do artigo. A. P. Ferreira e I. E. Mattos contribuíram na discussão e interpretação dos resultados e na revisão do texto. 


\section{Referências}

1. Secretaria de Vigilância em Saúde, Ministério da Saúde. Guia de vigilância epidemiológica. 7ạ Ed. Brasília: Ministério da Saúde; 2009.

2. Coura JR, Amaral RS. Epidemiological and control aspects of schistosomiasis in Brazilian endemic areas. Mem Inst Oswaldo Cruz 2004; 99 Suppl 1:13-9.

3. Quinino LRM, Costa JMBS, Aguiar LR, Wanderley TNG, Barbosa CS. Avaliação das atividades de rotina do Programa de Controle da Esquistossomose em municípios da Região Metropolitana do Recife, Pernambuco, entre 2003 e 2005. Epidemiol Serv Saúde 2009; 18:335-43.

4. Katz N, Peixoto SV. Análise crítica da estimativa do número de portadores de esquistossomose mansônica no Brasil. Rev Soc Bras Med Trop 2000; 33:303-8.

5. Secretaria de Vigilância em Saúde, Ministério da Saúde. Sistema Nacional de Vigilância em Saúde: relatório de situação, Maranhão. 2a Ed. Brasília: Ministério da Saúde; 2007.

6. Cutrim RMN. Aspectos clínicos e epidemiológicos da esquistossomose mansoni em três localidades da Baixada Ocidental Maranhense [Dissertação de Mestrado]. Rio de Janeiro: Instituto Oswaldo Cruz, Fundação Oswaldo Cruz; 1987.
7. Favre TC, Pieri OS, Barbosa CSB, Beck L. Avaliação das ações de controle da esquistossomose implementadas entre 1977 e 1996 na área endêmica de Pernambuco, Brasil. Rev Soc Bras Med Trop 2001; 34:569-76.

8. Secretaria de Vigilância em Saúde, Ministério da Saúde. Relatório de atividades do projeto de vigilância e monitoramento de endemias do convênio entre ENSP/FIOCRUZ e SVS/MS. Brasília: Ministério de Saúde; 2005.

9. Katz N, Chaves A, Pellegrino J. A simple device for quantitative stool thick-smear technique in Schistossomiasis mansoni. Rev Inst Med Trop São Paulo 1972; 13:51-6.

10. Farias LMM, Resende APC, Sabroza PC, SouzaSantos R. Análise preliminar do Sistema de Informação do Programa de Controle da Esquistossomose no período de 1999 a 2003. Cad Saúde Pública $2007 ; 23: 235-9$.

11. Gazzinelli A, Gazzinelli MF, Cadete MM, Pena Filho S, Sá IR, Kloos H. Sociocultural aspects of schistosomiasis mansoni in an endemic area in Minas Gerais, Brazil. Cad Saúde Pública 1998; 14:841-9.

Recebido em 14/Abr/2010

Versão final reapresentada em 03/Fev/2011 Aprovado em 18/Fev/2011 